\title{
RELACIÓN ENTRE LA EDUCACIÓN INCLUSIVA Y LA CALIDAD DE VIDA
}

\author{
Pablo Morilla Portela \\ Mari Carmen Pichardo Martínez \\ pichardo@ugr.es
}

Fecha de Recepción: 23 Febrero 2018

Fecha de Admisión: 10 Abril 2018

\section{RESUMEN}

La "Educación Inclusiva" supone una nueva forma de vivir la educación, mediante la cual, la totalidad de los sujetos tienen cabida en el sistema educativo y donde todos y cada uno de los alumnos, se enriquecen de esta diversidad. El presente estudio muestra como niños y niñas sin Necesidades Específicas de Apoyo Educativo (NEAE), escolarizados en un colegio con Alto Grado de Inclusión, muestran mayores niveles en la Calidad de Vida, así como en varias de sus dimensiones, en comparación con aquellos alumnos escolarizados en un sistema educativo tradicional. A través de esta investigación, también se demuestra como los alumnos que se han incorporado al centro educativo con Alto Grado de Inclusión, presentan mayores puntuaciones en la Calidad de Vida y sus diferentes dimensiones, cuando han pasado más tiempo experimentando esta inclusiva y natural forma de vivir la educación.

Palabras claves: educación inclusiva; escuela inclusiva; calidad de vida infantil; calidad de vida adolescente

\section{ABSTRACT}

Relationship between inclusive education and quality of live.

"Inclusive education" is a new way of living education, whereby, all the subjects have place in the educational system and where every student, is enriched by this diversity. This study shows how children without Special Educational Needs (SEN), enrolled in a school with High Degree of Inclusion, show higher levels in the Quality of Life as well as in several dimensions, compared to those students enrolled in a traditional educational system. Through this research, it is also shown how students who have joined the school with High Degree of Inclusion, have higher scores in the Quality of Life and its various dimensions, when they have spent more time experiencing this particular and natural way of living education.

Keywords: inclusive education; inclusion; children s quality of life; adolescents quality of life 


\section{RELACIÓN ENTRE LA EDUCACIÓN INCLUSIVA Y LA CALIDAD DE VIDA}

\section{ANTECEDENTES}

El mundo en el que vivimos tiende inevitablemente hacia la construcción de sociedades más diversas en las que sus habitantes convivan de forma armónica y pacífica, basándose no solo en el respecto y la aceptación de la diversidad, sino en la inclusión de la misma.

Los sistemas educativos de cada país tienen un papel muy importante que desempeñar, aunque la escuela, no puede ser el único agente que tome partido en este cambio mucho más global. Las instituciones educativas deben preparar a sus futuros ciudadanos, para poder afrontar y cumplir con sus derechos y deberes cívicos.

Como consecuencia de esta imperante demanda social, surge la necesidad de una nueva concepción de la educación y de la escuela, actualmente conocida como "Educación Inclusiva", un concepto mucho más amplio que su predecesor, "Integración Escolar", referido a alumnos con Necesidades Educativas Especiales (NEE). Esta nueva visión holística de la educación, pretende dar respuesta a la diversidad con la que los docentes se encuentran en las aulas, educando a todo el alumnado junto y construyendo una sociedad inclusiva, al mismo tiempo que se presta especial atención, a aquellos alumnos con Necesidades Específicas de Apoyo Educativo (NEAE) los cuales han sido tradicionalmente, objeto de exclusión en la escuela (Barrio de la Puente, 2009).

Esta nueva forma de entender la educación, ha probado ser un nivel superior en cuanto a humanidad y calidad, tanto para los posibles alumnos en riesgo de exclusión como para los que no, sin embargo, el concepto de Educación Inclusiva, es de difícil evaluación y no es fácil de objetivar. Desde la perspectiva del presente trabajo, la inclusión debe ser entendida como un continuo que posibilite a cada centro ubicarse en un punto único y diferente a todos los demás. Para hacer una evaluación objetiva sobre la situación en la que cada institución educativa se encuentra, se va a seguir la teoría propuesta por Dyson, Farrell, Polat, Hutcheson, y Gallannaugh (2004), según la cual, el criterio a tener en cuenta para valorar el grado de inclusión, es la proporción de alumnos con discapacidad. Un centro difícilmente puede ser inclusivo, cuando se niega la diversidad dentro de sus aulas.

No obstante y según esta nueva manera de educar, aunque la existencia de diversidad es quizás un criterio indispensable y posiblemente el más importante, este no debe ser el único parámetro que se establezca, para evaluar el grado de inclusión que se vive en un centro. Basándose en algunos de Ios principios de Booth y Ainscow (2011), y como concreta Muntaner (2013) a la premisa anterior (Presencia), el centro inclusivo debe cumplir con otras dos: Participación (de todos los alumnos en las actividades, experiencias y situaciones y en la vida escolar) y Progreso (entendido como la implantación de una educación de calidad y adaptada a las necesidades del alumno, a través de la cual los alumnos progresan y aprenden.

Es posible observar, numerosas investigaciones que demuestran los beneficios de una Educación Inclusiva en alumnos con riesgo de exclusión, desde diferentes perspectivas: tanto desde el ámbito social (Ntshangase, Mdikana y Cronk, 2008; Reed, McIntyre, Dusek, y Quintero, 2011), como desde el académico (Daniel y King, 1997; Rollins, 2007).

Sin embargo, la Educación Inclusiva, supone algo mucho mayor que una serie de beneficios para aquellos alumnos con discapacidad. Una escuela inclusiva, sumerge a los chicos y chicas en una vivencia íntima y un aprendizaje intrínseco de lo que significa, supone y conlleva, "ser diferente". Aunque en menor medida, diversos autores han estudiado los beneficios que supone para aquellos alumnos, que aun siendo singulares y únicos, no precisan de NEAE por parte del profesorado. Contradiciendo los trabajos de diversos autores (Huber, Rosenfeld, y Fiorello, 2001; Staub y Peck, 1994), McDonnell et al. (2003) probaron como no existían diferencias en los logros académicos por parte de los alumnos sin NEAE en contextos inclusivos. Desde el punto de vista social, numerosos 
trabajos han probado las ventajas de entornos inclusivos (Kalyva y Agaliotis, 2009; Siperstein, Parker, Bardon, y Widaman, 2007; Slininger, Sherrill, y Jankowski, 2000). Los resultados de dichos estudios sostienen la teoría de Boer, Pijl y Minnaert (2013), quienes relacionan actitudes positivas de los alumnos hacia sus compañeros con discapacidad, con el grado de participación e interacción social.

Uno de los conceptos que sirve como referencia y guía conceptual para emprender cambios y mejoras en la escuela en su proceso hacia la Educación Inclusiva es el constructo de Calidad de Vida (Muntaner, 2013). Este puede ser definido como un estado deseado de bienestar personal que es multidimensional, tiene propiedades éticas - universales- y émicas -ligadas a la cultura-, tiene componentes objetivos y subjetivos, y está influenciada por factores personales y ambientales (Schalock y Verdugo, 2007). Como recogen Ruiz, Gomez-Vela, Fernández y Badía (2014) dicho concepto surge como guía y criterio para evaluar las estrategias de mejora y las buenas prácticas educativas (Gómez-Vela y Verdugo, 2009; Muntaner, Forteza, Rosselló, Verger y De la Iglesia, 2010).

\section{OBJETIVOS}

El objetivo del presente estudio se centrará en la Calidad de Vida, tanto infantil, como adolescente, evidenciando si hay diferencias significativas en la Calidad de Vida (o en alguna de sus múltiples dimensiones) de los niños y niñas sin NEAE que viven una experiencia educativa más inclusiva (Alto Grado de Inclusión), respecto a aquellos que se encuentran escolarizados en un modelo tradicional y menos inclusivo (Bajo Grado de Inclusión). Se profundizará también en el modelo de Educación Inclusiva, analizando si existen modificaciones significativas en la Calidad de Vida, a medida que los alumnos pasan más años enriqueciéndose de esta diversidad.

\section{PARTICIPANTES}

En la presente investigación participaron un total de cinco instituciones educativas y se contó con 2.056 sujetos, de los cuales, 290 no fueron seleccionados por los siguientes motivos: tener alguna discapacidad o dificultad de aprendizaje, ser alumno perteneciente al censo de NEAE, tener una Adaptación Curricular Individualizada, cursar un nivel curricular inferior, contestar incorrectamente a las preguntas de control, o haber obtenido puntuaciones inapropiadas en la Escala de Deseabilidad Social o Aquiescencia en el Cuestionario de Evaluación de la Calidad de Vida de alumnos Adolescentes (CCVA). Tras esta selección, la muestra final fue de 1.766 sujetos, de los cuales, 758 pertenecían a la Educación Primaria Obligatoria (EPO) y 1.008 a la Educación Secundaria Obligatoria (ESO), abarcando los cursos desde $3^{\circ}$ de EPO hasta 6ำ de EPO y desde $1^{\circ}$ de ESO hasta $4^{\circ}$ de ESO.

Los 758 sujetos pertenecientes a la EPO, (386 chicos y 370 chicas), tenían edades comprendidas entre 8 y 13 años $(M=9,75$; DT =1,20), eran procedentes de 4 centros: 1 público $(17,8 \%)$ y 3 concertados (82,2\%); encontrándose un $26,9 \%$ matriculado en un centro inclusivo y un $73,1 \%$ en otros centros considerados no inclusivos. El 24,5\% cursaban 3을 de EPO; el 26,5\%, $4^{\circ}$ de EPO; el $22,5 \%, 5^{\circ}$ de EPO y el 26,5\%, 60 de EPO.

En cuanto a los 1.008 alumnos pertenecientes a la ESO, (476 chicos y 507 chicas), sus edades oscilaban entre los 12 y los 18 años ( $M=13,88$; DT =1,31), los cuales habían sido seleccionados de 4 centros: 1 público y 3 concertados (17,2\% y 82,8\% respectivamente); siendo el porcentaje de alumnos del centro inclusivo un $21,8 \%$, frente al $78,2 \%$ que pertenecían a centros no inclusivos. El $25,7 \%$ estaban cursando $1^{\circ}$ de ESO; el 25,5\%, $2^{\circ}$ de ESO; el 24,9\%, 3ํㅡㄹ de ESO; y el 23,9\%, $4^{\circ}$ de ESO. 


\section{RELACIÓN ENTRE LA EDUCACIÓN INCLUSIVA Y LA CALIDAD DE VIDA}

\section{MÉTODO}

\section{Instrumentos:}

En la investigación se utilizaron dos cuestionarios para evaluar la Calidad de Vida:

El primer instrumento fue el Cuestionario de Evaluación de la Calidad de Vida en la Infancia (CVI) de Sabeh, Verdugo, Prieto y Contini (2009). El cuestionario evalúa el bienestar de la población infantil, con y sin necesidades especiales, en las siguientes dimensiones: relaciones interpersonales, desarrollo personal, bienestar emocional, bienestar físico y bienestar material.

Los resultados del análisis de fiabilidad, mediante el alfa de Cronbach fueron los siguientes: Bienestar Emocional, .80; Relaciones Interpersonales, .74; Desarrollo Personal y de Actividades, .60; Bienestar Físico, .55; Bienestar Material, .67; y Calidad de Vida Total, .90.

El segundo instrumento fue el CCVA (Gomez-Vela y Verdugo, 2009). Dicho instrumento consta de 61 ítems y evalúa las siguientes dimensiones: Relaciones interpersonales, Bienestar material, Desarrollo personal, Bienestar emocional, Integración en la comunidad, Bienestar físico y Autodeterminación. Dentro de los 61 Ítems incluyen siete pares de contenido inverso para controIar la aquiescencia, cumpliendo con las recomendaciones de numerosos autores (Antaki y Rapley, 1996; Kilsby, Bennert y Beyer, 2002; Ramirez, 2005), y otros 10 ítems para controlar la deseabilidad social (Antaki y Rapley, 1996; Schwarz, 1999). Los ítems consisten en enunciados sencillos ante los que el adolescente debe expresar su grado de acuerdo o desacuerdo. El formato de respuesta es tipo Likert con cuatro alternativas. La puntuación asignada a cada categoría oscila entre 1 y 4 puntos (1 significa "Total desacuerdo" y 4 "Total acuerdo").

El cuestionario presenta una fiabilidad adecuada, siendo similares los coeficientes de consistencia interna de la investigación actual, una vez contrastados con los resultantes en el diseño y validación del cuestionario. Los datos obtenidos por Gomez-Vela y Verdugo (2009) comparados con los de la presente investigación (Alfa de Cronbach), son los que siguen respectivamente: Bienestar Emocional, .82/.81; Integración / Presencia en la comunidad, .61/.76; Relaciones Interpersonales, .67/.71; Desarrollo Personal, .65/.63; Bienestar Físico, .58/.49; Autodeterminación, .58/.56; Bienestar Material, .58/.60; y Calidad de Vida Total, .84/.89.

\section{Procedimiento:}

Para la obtención de la muestra se seleccionó inicialmente, un centro al que se ha catalogado con un Alto Grado de Inclusión, con base en el cumplimiento de estos tres principios: En primer lugar (Presencia), el centro contaba con un $11,33 \%$ de alumnos oficialmente inscritos en el Censo de NEAE y con un $17 \%$ de sujetos que recibía algún tipo de medida de atención a la diversidad. En segundo lugar (Participación), por un lado se analizó el total de las actividades evaluadas por los docentes del centro durante el último curso académico, habiendo participado el $100 \%$ de los alumnos en todas y cada una de ellas; por otro lado, mediante visitas al colegio se comprobó cómo los estudiantes participan activamente en la vida comunitaria y escolar. Por último (Progreso) se corroboró que el $94 \%$ de alumnos era receptor y se beneficiaba de diferentes medidas de atención a la diversidad si cumplía con alguna de las siguientes características: estar oficialmente inscrito en censo de NEAE, tener discapacidad, ser alumno de altas capacidades o haber experimentado una incorporación tardía al sistema educativo.

A continuación, se propuso participar en la investigación a todos los centros educativos que se encontraban en un radio de un kilómetro, aproximadamente, de dicho centro. Se invitó individualmente a que la totalidad de centros participara en la investigación. Cuatro centros más, accedieron a participar, presentando un Bajo Grado de Inclusión al no cumplir el principal y más básico de los 
parámetros: contar con un alumnado que ofrezca y proporcione esa diversidad (Presencia), siendo $0,5 \%$ del total de alumnos, los que estaban inscritos en el censo de NEAE. Además, dentro de este pequeño porcentaje, tan solo el $41 \%$ de alumnos que estaban oficialmente inscritos en el censo de NEAE, tenían discapacidad, eran alumnos de altas capacidades o habían experimentado una incorporación tardía al sistema educativo; era receptor y se beneficiaba de diferentes medidas de atención a la diversidad (Progreso).

Tras obtener la autorización de los cinco centros para llevar a cabo el actual trabajo, se envió una propuesta o modelo de autorización a los mismos, de forma que todos los sujetos que participaran en la investigación, tuvieran el consentimiento de sus padres.

Tras la aceptación de las familias y centros, dos encuestadores debidamente formados administraron los cuestionarios de forma colectiva, clase por clase. El tiempo aproximado de contestación de cada uno de los cuestionarios fue de unos 15-30 minutos. Obtenidos los datos, éstos se analizaron a través del programa estadístico SPSS- versión 11.0 para Windows. Para el análisis de los datos se realizaron las pruebas de diferencia de medias (t de student) más adecuadas en función de la homogeneidad de las varianzas.

\section{RESULTADOS}

La tabla 1 muestra la existencia de diferencias significativas entre los centros de Alto y Bajo Grado de Inclusión en el Bienestar Emocional, en las Relaciones Interpersonales, en el Bienestar Material, en el Desarrollo Personal y Actividades y en la Calidad de Vida, que correspondería a la suma de todas las dimensiones. Únicamente, no se encuentran diferencias significativas en el Bienestar Físico. En todas las variables de calidad de vida analizadas los participantes de las escuelas con Alto Grado de Inclusión obtienen puntuaciones significativamente más elevadas que sus compañeros procedentes de escuelas con Bajo Grado de Inclusión.

Tabla 1

Diferencias en la Calidad de Vida Infantil en función del Grado de Inclusión

\begin{tabular}{lcccccc}
\hline & $\begin{array}{c}\text { Grado de } \\
\text { Inclusión }\end{array}$ & $\mathrm{N}$ & Media & $\begin{array}{c}\text { Desviación } \\
\text { típ. }\end{array}$ & $\mathrm{t}$ & $\mathrm{p}$ \\
\hline Bienestar & Bajo & 501 & 3.26 & .481 & -3.21 & .001 \\
Emocional & Alto & 196 & 3.38 & .384 & & \\
$\begin{array}{l}\text { Relaciones } \\
\text { interpersonales }\end{array}$ & Bajo & 475 & 3.35 & .403 & -3.56 & .000 \\
Desarrollo & Alto & 197 & 3.45 & .320 & & \\
Personal & Bajo & 500 & 3.16 & .434 & -2.37 & .018 \\
Actividades & Alto & 199 & 3.24 & .370 & & \\
Bienestar Físico & Bajo & 518 & 3.39 & .456 & -1.14 & .256 \\
& Alto & 200 & 3.43 & .404 & & \\
Bienestar & Bajo & 528 & 3.28 & .477 & -3.46 & .001 \\
Material & Alto & 202 & 3.40 & .390 & & \\
Calidad de Vida & Bajo & 404 & 3.32 & .360 & -2.37 & .018 \\
(Total) & Alto & 187 & 3.39 & .278 & & \\
\hline
\end{tabular}

En cuanto a los resultados de los alumnos que cursan la ESO (Tabla 2), encontramos diferencias muy significativas en el Bienestar Físico. Las diferencias son significativas en cuanto a la Autodeterminación y a la Calidad de Vida. El resto de dimensiones no muestran diferencias significativas. En todas las variables que resultaron significativas, las mayores puntuaciones fueron obtenidas por el alumnado del colegio con Alto Grado de Inclusión. 


\section{RELACIÓN ENTRE LA EDUCACIÓN INCLUSIVA Y LA CALIDAD DE VIDA}

Tabla 2

Diferencias en la Calidad de Vida Adolescente en función del Grado de Inclusión.

\begin{tabular}{lcccccc}
\hline & $\begin{array}{c}\text { Grado de } \\
\text { inclusión }\end{array}$ & $\mathrm{N}$ & Media & $\begin{array}{c}\text { Desviación } \\
\text { típ. }\end{array}$ & $\mathrm{t}$ & $\mathrm{P}$ \\
\hline Bienestar Emocional & Bajo & 457 & 3.08 & .532 & -.874 & .382 \\
& Alto & 120 & 3.13 & .508 & & \\
Integración & Bajo & 452 & 2.85 & .456 & -.349 & .727 \\
& Alto & 122 & 2.87 & .444 & & \\
Relaciones & Bajo & 442 & 3.48 & .360 & -1.57 & .116 \\
Interpersonales & Alto & 122 & 3.53 & .331 & & \\
Desarrollo Personal & Bajo & 461 & 3.30 & .404 & -1.59 & .112 \\
& Alto & 123 & 3.36 & .366 & & \\
Bienestar Físico & Bajo & 467 & 3.54 & .408 & -2.38 & .018 \\
Autodeterminación & Alto & 121 & 3.67 & .358 & & \\
& Bajo & 448 & 3.09 & .404 & -1.85 & .065 \\
Bienestar Material & Alto & 122 & 3.16 & .397 & & \\
Bajo & 455 & 2.84 & .509 & -1.66 & .097 \\
Calidad de Vida & Alto & 123 & 2.92 & .535 & & \\
(Total) & Bajo & 344 & 3.15 & .273 & -1.84 & .066 \\
& Alto & 115 & 3.21 & .261 & & \\
\hline
\end{tabular}

Si se lleva a cabo un análisis de los sujetos escolarizados en un centro con Alto Grado de Inclusión, se puede apreciar como existen diferencias significativas tanto en diferentes dimensiones, como en el conjunto Calidad de Vida, en función del número de años que los alumnos han pasado escolarizados en el modelo de Educación Inclusiva (por un lado, se diferencia entre alumnos que han estado matriculados de 1 a 3 años y por otro, alumnos que han estado matriculados 4 años 0 más).

A medida que los sujetos han estado más años en el centro, los niños y niñas de EPO evidencian niveles significativamente superiores de Bienestar Emocional, las Relaciones Interpersonales, el Bienestar Físico y la Calidad de Vida Total (ver Tabla 3).

Tabla 3

Diferencias en la Calidad de Vida Infantil en función del no de años en un centro con Alto Grado de Inclusión

\begin{tabular}{lcccccc}
\hline & $\begin{array}{c}\text { Años en el } \\
\text { centro }\end{array}$ & $\mathrm{N}$ & Media & $\begin{array}{c}\text { Desviación } \\
\text { típ. }\end{array}$ & $\mathrm{t}$ & $\mathrm{p}$ \\
\hline Bienestar Emocional & $1-3$ & 29 & 3.21 & .378 & 2.50 & .013 \\
& 4 o más & 167 & 3.40 & .379 & & \\
Relaciones & $1-3$ & 31 & 3.30 & .368 & 3.03 & .003 \\
interpersonales & 4 o más & 166 & 3.48 & .303 & & \\
Desarrollo Personal & $1-3$ & 30 & 3.15 & .374 & 1.42 & .158 \\
y Actividades & 4 o más & 169 & 3.26 & .368 & & \\
Bienestar Físico & $1-3$ & 31 & 3.28 & .345 & 2.31 & .022 \\
& 4 o más & 169 & 3.46 & .409 & & \\
Bienestar Material & $1-3$ & 31 & 3.39 & .343 & -.81 & .936 \\
& 4 o más & 171 & 3.40 & .400 & & \\
Calidad de Vida & $1-3$ & 28 & 3.24 & .287 & 3.03 & .003 \\
(Total) & 4 o más & 159 & 3.41 & .269 & & \\
\hline
\end{tabular}

En lo que a los alumnos de la ESO se refiere (Tabla 4), las dimensiones que reflejan diferencias significativas en alumnos que han estado mayor tiempo escolarizados son el Bienestar Emocional, el Desarrollo Personal y el Bienestar Físico, así como la Calidad de Vida. 
Tabla 4

Diferencias en la Calidad de Vida Adolescente en función del $n^{\circ}$ de años en un centro con Alto Grado de Inclusión

\begin{tabular}{lcccccc}
\hline & $\begin{array}{c}\text { Número de } \\
\text { años }\end{array}$ & $\mathrm{N}$ & Media & $\begin{array}{c}\text { Desviación } \\
\text { típ. }\end{array}$ & $\mathrm{T}$ & $\mathrm{P}$ \\
\hline Bienestar Emocional & $1-3$ & 12 & 2.94 & .560 & -1.35 & .177 \\
& 4 o más & 108 & 3.15 & .500 & & \\
Integración & $1-3$ & 13 & 2.77 & .359 & -.799 & .426 \\
& 4 o más & 109 & 2.88 & .453 & & \\
Relaciones & $1-3$ & 13 & 3.45 & .263 & -1.01 & .316 \\
Interpersonales & 4 o más & 109 & 3.54 & .339 & & \\
Desarrollo Personal & $1-3$ & 13 & 3.14 & .267 & -2.31 & .022 \\
& 4 o más & 110 & 3.39 & .369 & & \\
Bienestar Físico & $1-3$ & 13 & 3.47 & .491 & -1.95 & .054 \\
& 4 o más & 108 & 3.65 & .335 & & \\
Autodeterminación & $1-3$ & 13 & 3.32 & .505 & 1.19 & .256 \\
& 4 o más & 109 & 3.15 & .380 & & \\
Bienestar Material & $1-3$ & 13 & 2.78 & .465 & -1.02 & .310 \\
& 4 o más & 110 & 2.94 & .541 & & \\
Calidad de Vida & $1-3$ & 12 & 3.13 & .173 & -1.12 & .266 \\
(Total) & 4 o más & 103 & 3.22 & .269 & & \\
\hline
\end{tabular}

\section{CONCLUSIONES}

Los resultados de este estudio, en relación con el objetivo principal de la investigación, indican que los alumnos sin NEAE que experimentan y viven un modelo de Escuela Inclusiva tienen grados más altos de Calidad de Vida, tanto en la EPO como en la ESO. Haciendo un análisis de los sujetos inmersos en modelos educativos con un Alto Grado de Inclusión se demuestra cómo, a medida que los sujetos han pasado más tiempo en este tipo de entornos, se obtienen niveles más Altos de Calidad de Vida en las dos etapas educativas objeto de estudio.

Un análisis más profundo muestra que los alumnos de EPO pertenecientes al centro con Alto Grado de Inclusión, no solo muestran niveles superiores en cuanto a la Calidad de Vida, sino a todas sus dimensiones (exceptuando la de Bienestar Físico), es decir: Bienestar Emocional, Relaciones Interpersonales, Desarrollo Personal y Actividades y Bienestar Material. Los alumnos de la ESO, además de mostrar niveles superiores de Calidad de Vida, también lo hacen en cuanto a Bienestar Físico y Autodeterminación.

Atendiendo al tiempo que los sujetos han estado escolarizados en el centro con Alto Grado de Inclusión, en la EPO se muestran puntuaciones más altas en cuanto a Calidad de Vida, así como en Bienestar Emocional, Bienestar Físico y Relaciones Interpersonales. Los alumnos de la ESO que han estado más tiempo en el centro, también obtienen puntuaciones más altas en Calidad de vida, pero las dimensiones varían ligeramente: Bienestar Emocional, Bienestar Físico y Desarrollo Personal.

Estas puntuaciones más elevadas, podrían ser la consecuencia de una serie de políticas educativas más inclusivas tal y como se refleja en el Index for Inclusion (Booth y Ainscow, 2011).

Las puntuaciones medias resultantes de la Calidad de Vida y sus dimensiones en la EPO, son similares a las que se desprenden de otras investigaciones como Ruiz, Gomez-Vela, Fernández y Badía (2014). En cuanto a los alumnos de la ESO, es posible observar como las puntuaciones son similares a las de Gomez-Vela y Verdugo (2009), cuyos resultados son superiores a 75 puntos porcentuales en todas las escalas. Utilizando este tipo de puntuación, los alumnos del modelo de Alto Grado de Inclusión muestran puntuaciones por encima de 74.3 en todas las dimensiones, salvo en 


\section{RELACIÓN ENTRE LA EDUCACIÓN INCLUSIVA Y LA CALIDAD DE VIDA}

la de Bienestar Material. Dicha diferencia podría explicarse por dos cuestiones: la primera sería el cambio que se ha producido en términos macroeconómicos en España desde la recogida de datos del trabajo anterior hasta ahora y la segunda, podría ser el tipo de muestra que se ha utilizado para dicha investigación: 79 alumnos de centros concertados, 333 de centros públicos y 709 de centros privados en los que se presupone un nivel adquisitivo mayor de las familias.

Antes de concluir en el presente estudio deben reconocerse ciertas limitaciones: en primer lugar, la muestra no pudo ser seleccionada de forma aleatoria y pertenece a un zona geográfica muy concreta; en segundo, por motivos de escolarización, la muestra que se ha utilizado, a la hora de analizar la Calidad de Vida en función del número de años que los alumnos habían cursado en el modelo de Educación Inclusiva, es muy inferior en aquellos alumnos que llevan entre 1 y 3 años, respecto a aquellos que han estado más de 4 años; y por último, tal y como señalan Gomez-Vela, Verdugo y González (2014), la fiabilidad de alguna de las dimensiones del CCVA, pueden no haber sido suficientemente altas. En futuras investigaciones, sería interesante poder ver si existe relación entre la Calidad de Vida y otros constructos, en alumnos con y sin discapacidad, tales como la autoestima, el autoconcepto, la conducta prosocial o la empatía. Aspectos tan importantes pueden estar relacionados con esta nueva manera de entender la "Educación Inclusiva", una herramienta y un proceso de obligada necesidad si se pretende alcanzar y construir "Sociedades Inclusivas".

\section{BIBLIOGRAFÍA}

Antaki, C. y Rapley, M. (1996). Questions and answers to psychological assessment schedules: Hidden troubles in "quality of life" interviews. Journal of Intellectual Disability Research, 40, 421-437.

Barrio de la Puente, J. L. (2009) Hacia una Educación Inclusiva para todos. Revista Complutense de Educación, 20 (1), 13-31.

De Boer, A., Pijl, S. J., y Minnaert, A., (2012) Students' Attitudes towards Peers with Disabilities: A review of the literature. International Journal of Disability, Development and Education, 59 (4), 379-392.

Booth, T. y Ainscow, M. (2011). Index for inclusion: Developing learning and participation in schools ( $3^{\text {rd }}$ ed.). Bristol, England: Centre for Studies on Inclusive Education.

Daniel, L. G. y King, D. A. (1997). Impact of inclusion education on academic achievement, student behavior and self-esteem, and parental attitudes. Journal of Educational Research, 91(2), 67-80.

Dyson, A., Farrell, P., Polat, F., Hutcheson, G., y Gallannaugh, F. (2004). Inclusion and pupil Achievement. (London, DfES).

Gomez-Vela, M. y Verdugo, M. A. (2009) Cuestionario de evaluación de la calidad de vida de alumnos adolescentes: manual de aplicación. Madrid: CEPE.

Huber, K. D., Rosenfeld, J. G., y Fiorello, C. A. (2001). The differential impact of inclusion and inclusive practices on high, average and low-achieving general education students. Psychology in the Schools, 38, 497-504.

Kalyva, E., y Agaliotis, I. (2009). Can contact affect Greek children's understanding of and attitudes towards peers with physical disabilities? European Journal of Special Needs Education, 24, 213-220.

Kilsby, M., Bennert, K. y Beyer, S. (2002). Measuring and reducting acquiescence in vocational profiling procedures for first time job-seeketrs with mental retardation. Journal of Vocational Rehabilitation, 17 (4), 287-299.

McDonnell, J., Thorson, N., Disher, S., Mathot-Buckner, C., Mendel, J., y Ray, L. (2003). The achievement of students with developmental disabilities and their peers without disabilities in 
inclusive settings: an exploratory study. Education and Treatment of Children, 26(3), 224-236.

Muntaner Guasp, J. J. (2013). Calidad de vida en la escuela inclusiva. Revista ibero-americana de educação, 63, 35-49.

Muntaner, J. J., Forteza M. D., Rosselló M. R., Verger S., y De la Iglesia B. (2010). Estándares e indicadores para analizar la calidad de vida del alumnado con discapacidad durante su proceso educativo. Mallorca: Edicions UIB.

Ntshangase, S., Mdikana, A., y Cronk, C. (2008). A comparative study of self-esteem of adolescent boys with and without learning disabilities. International Journal of Special Education, 23(2), 7584

Ramirez, S. (2005). Evaluation Acquiescence to Yes-No Questions in fear assessment of children with and without mental retardation. Journal of Developmental and Physical Disabilities, 17 (4), 337-343.

Reed, F.D., McIntyre, L.L., Dusek, J. y Quintero, N. (2011). Preliminary assessment of friendship, problem behavior, and social adjustment in children with disabilities in an inclusive education setting. Journal of Developmental and Physical Disabilities, 23(6), 477-489.

Rollins, L. M. (2007). The influence of full inclusion on academics and self-concepts of students with learning disabilities. (Tesis doctoral no publicada). Walden Univesity Minnesota.

Ruiz Fernandez, B., Gomez-Vela, M., Fernández Pulido, R., y Badía Corbella, M. (2014). Revista Iberoamericana De Educación, 66, 59-74.

Sabeh, E. N., Verdugo, M.A., Prieto, G. y Contini, N.E. (2009). CVI-CVIP. Cuestionarios de evaluación de la calidad de vida en la infancia. Madrid: CEPE.

Schwarz, N. (1999). Self-reports: How the questions shape the answers. American Psychologist, 54, 93-105.

Schalock, R. L. (1997). Evaluación de programes sociales. Siglo Cero, 28 (2), 13-36.

Schalock, R. L. y Verdugo, M. A. (2007). El concepto de calidad de vida en los servicios y apoyos para personas con discapacidad intelectual. Siglo Cero, 224, 21-36.

Siperstein, G. N., Parker, R. C., Bardon, J. N., y Widaman, K. F. (2007). A national study of youth attitudes toward the inclusion of students with intellectual disabilities. Exceptional Children, 73, $435-455$.

Slininger, D., Sherrill, C., y Jankowski, C. M. (2000). Children's attitudes toward peers with severe disabilities: Revisiting contact theory. Adapted Physical Activity Quarterly, 17, 176-196.

Staub, D. y Peck, C. A. (1994). What are the outcomes for non-disabled students? Educational Leadership, 54(4), 36-40.

Verdugo, M. A. (2009). El cambio educativo desde una perspectiva de calidad de vida. Revista de Educación, 349, 23-43. 
